\title{
Ergonomic issues in ewe cheese production: reliability of the Strain Index and OCRA Checklist risk assessments
}

\author{
John Rosecrance, ${ }^{1}$ Robert Paulsen, ${ }^{1}$ David Gilkey, ${ }^{1}$ Lelia Murgia, ${ }^{2}$ Thomas Gall² \\ ${ }^{1}$ College of Veterinary Medicine and Biomedical Sciences, Colorado State University, USA; \\ ${ }^{2}$ Department of Agraria, University of Sassari, Italy
}

\begin{abstract}
Occupational ergonomists often use a variety of methods to identify jobs that are considered at high risk for the development of work-related musculoskeletal illnesses. The Strain Index (SI) and the Occupational Repetitive Actions (OCRA) Checklist are two popular upper limb risk assessment tools used in many industries, including the agro-food industry. Both methods are based on similar biomechanical, physiological and epidemiologic principles, but their approach to quantification and estimation of risk factor magnitude is quite different. The purpose of this study was to assess the inter-method reliability of SI and OCRA Checklist. Methods: Twenty-one jobs were video recorded in a Sardinian cheese manufacturing facility. Eight raters were recruited to assess job exposures to physical risk factors using the SI and OCRA Checklist. Inter-method reliability was characterized using proportion of overall agreement, Cohen's kappa, and Spearman and Pearson correlations. Results: Strain Index and the OCRA Checklist assessments produced generally reliable results, classifying the risk of 35 of 42 (83\%) job exposures similarly. Conclusions: The OCRA Checklist and SI risk assessments are reliable upper limb measures of physical work exposures. Both measures appear useful for assessing risk of upper limb disorders of work tasks in the agro-food industry. However, the SI is specific to disorders of the distal upper limb and perhaps most useful for assessing risk in work primarily involving the wrist and fingers. Whereas the OCRA Checklist, which
\end{abstract}

Correspondence: Robert Paulsen, 1681 Campus Delivery, Colorado State University. Fort Collins, Colorado, USA 80523.

Tel. +001.970.491.1405.

E-mail: paulsen.rj@gmail.com

Key words: strain index, OCRA checklist, inter-method reliability, musculoskeletal disorders, risk assessment methods

Acknowledgments: the present study was supported by the Center for Disease Control (CDC) / National Institute for Occupational Safety and Health (NIOSH) Mountain and Plains Education and Research Center (grant number: 5T420H009229-06). Its contents are solely the responsibility of the authors and do not necessarily represent the official views of the CDC or NIOSH.

@C Copyright J. Rosecrance et al., 2013

Licensee PAGEPress, Italy

Journal of Agricultural Engineering 2013; XLIV(s2):e139

doi:10.4081/jae.2013.s2.e139

This article is distributed under the terms of the Creative Commons Attribution Noncommercial License (by-nc 3.0) which permits any noncommercial use, distribution, and reproduction in any medium, provided the original author(s) and source are credited. includes an assessment of the shoulder, may be more appropriate for evaluating jobs that also require extended periods of reaching and shoulder activity.

\section{Introduction}

The Strain Index (SI) (Moore and Garg 1995) and the Occupational Repetitive Actions (OCRA) Checklist (Colombini et al., 2000; Colombini et al., 2011) are two of the most comprehensive and widely used tools used to quantify exposure to physical risk factors for upper extremity musculoskeletal disorders (MSDs). The SI and OCRA Checklist are semi-quantitative observational assessment tools employed by occupational health practitioners and physicians to systematically evaluate ergonomic job hazards in the agro-food industry. Both methods produce a composite risk index that summarizes the magnitude of physical risk factor exposure. The results of an SI or OCRA Checklist assessment are usually interpreted according to tiered risk classification criteria, which usually categorize risk indexes as no risk, moderate risk, or high risk. These categorical risk classifications can be used to map the ergonomic hazards within a facility or a department, to identify the impact of interventions, and to compare an observational risk assessment with other risk assessment methods.

The purpose of this study was to investigate whether the SI and OCRA Checklist would reliably assess risk when they are used to evaluate the same job exposures (inter-method reliability). The SI and OCRA Checklist were used to evaluate physical job exposures to the left and right upper limbs of workers performing 21 different cheese manufacturing jobs.

\section{Methods}

The SI is an observational assessment tool used to quantify exposures related to the development of general distal upper extremity MSDs (Moore and Garg 1995). The SI risk index is the product of six task-variable scores determined by an SI rater: (i) intensity of exertion, (ii) duration of exertion, (iii) efforts per minute, (iv) hand/wrist posture, (v) speed of work, and (vi) task duration per day (Moore and Garg 1995).

Similar to the SI, the OCRA Checklist is an observational assessment tool, but it quantifies exposure to physical risk factors affecting the entire upper extremity, including the shoulder. The OCRA Checklist also summarizes exposure in terms of six task-variable scores (i) frequency of technical actions, (ii) force, (iii) awkward postures and movements, (iv) additional factors, (v) lack of sufficient recovery, and (vi) task duration (Colombini et al., 2002; Colombini et al., 2011).

Exposures to physical risk factors for upper extremity MSDs were assessed for 21 cheese manufacturing jobs performed at a facility in 
Sardinia Italy. These 21 jobs included all of the major stages of Pecorino Romano production, from draining curd to product packaging. Operators performing these jobs were required to exert a variety of repetitive upper extremity movements and forceful exertions. Handheld digital video camcorders were used to record at least five work-cycles of upper limb activity in the frontal and sagittal planes. Job and break/recovery duration information was obtained by direct observation and onsite interviews.

Eight individuals rated the left and right limb exposures for operators performing 21 cheese manufacturing jobs. Regardless of prior experience, raters received structured SI and OCRA Checklist training before evaluating any jobs for the present study.

Raters analyzed videos of each cheese manufacturing job using the SI and OCRA Checklist according to the instructions of the developers of each method (Moore and Garg 1995; Colombini et al., 2011). Raters waited at least three weeks after rating all of the jobs with one method before reevaluating them with the other method.

\section{Data analysis and statistical methods}

Strain Index and OCRA Checklist risk indexes, which are continuous variables, can only be compared by categorizing them using common exposure classification criteria (also referred to as hazard thresholds or cut-points). These criteria provide a simple summary of the physical risk factor exposure magnitude experienced by the worker. The exposure classifications chosen for inter-method reliability analyses are shown in Table 1, and they were similar to those used in previous SI and OCRA Checklist or OCRA Index studies (Apostoli et al., 2004; Stevens et al., 2004; Stephens et al., 2006; Spielholz et al., 2008; Jones and Kumar 2010; Colombini et al., 2011; Chiasson et al., 2012).

Inter-method reliability analyses of SI and OCRA Checklist exposure classifications were performed using mean risk indexes based on the eight replicate assessments made of each job (one assessment per rater using both the SI and the OCRA Checklist). The proportion of overall agreement $\left(p_{o}\right)$ for these mean risk indexes was calculated, and Cohen's weighted kappa coefficient using Fleiss-Cohen weights $\left(\kappa_{w}\right)$ characterized the chance-corrected agreement between both measures. To assist with the interpretation of results, Landis and Koch's (1977) verbal criteria of kappa coefficient magnitude were employed: $\kappa_{w}<0.20$, poor or slight agreement; $0.21 \leq \kappa_{w} \leq 0.40$, fair agreement; $0.41 \leq \kappa_{w} \leq 0.60$, moderate agreement; $0.61 \leq \kappa_{w} \leq 0.80$, substantial agreement; and $\kappa_{w}>0.80$, almost perfect agreement. Spearman's rankorder correlation coefficient $\left(r_{s}\right)$ was also calculated to represent the strength of association between risk classifications. Additionally, the linear relationship between SI and OCRA checklist continuous risk indexes was characterized by Perason's product-moment correlation coefficient $(r)$. All statistical analyses were completed using SAS/STAT® Software (SAS Institute, Cary, NC) version 9.3 (2012) on a Windows PC. Confidence intervals were calculated with $95 \%$ certainty.

\section{Results and discussion}

Jobs were predominantly machine-paced and had a mean cycle time of 41.5 seconds $( \pm 31.2)$. The mean risk index for all job exposures was 22.2 for the SI assessments and 19.2 for the OCRA Checklist assessments.

Table 2 displays the distribution of SI and OCRA Checklist risk classifications for the 42 cheese manufacturing exposures (21 jobs $\mathrm{x} 2$ limbs exposed per job). The two methods attributed the same level of risk to 35 of the 42 job exposures $\left(p_{0}=83.3 \%\right)$. Table 2 also shows that the risk classification for nearly all of the exposures was ranked as "moderate risk" or "high risk" (97.6\% for the SI and 92.9\% for the OCRA Checklist). The SI ranked more jobs as high risk (61.9\%) than did the OCRA Checklist (50.0\%). Further, all 7 of the risk classification disagreements were biased toward higher SI risk assessments.

The relatively high level of overall agreement was also substantially greater than would be predicted by chance alone. Cohen's weighted kappa coefficient was 0.76 (95\% CI: 0.61-0.92). The lower limit of the $\kappa_{w} 95 \%$ confidence interval was equal to the lower criterion boundary of what Landis and Koch termed "substantial agreement" in ratings beyond chance. The Spearman rank-order correlation coefficient $\left(r_{s}\right)$ for the 42 pairs of risk classifications was 0.80 (95\% CI: 0.66-0.89). This strong correlation provides further support for the good inter-method reliability of the SI and OCRA Checklist.

The linear relationship between the SI and OCRA Checklist risk indexes was very strong. For the pairs of 42 continuous risk indexes, the Pearson product-moment correlation coefficient $(r)$ was $0.94(95 \%$ CI: 0.89-0.97). Figure 1 displays this relationship between risk indexes.

The present study appears to suggest a greater inter-method reliability between SI and OCRA Checklist assessments than previous studies have reported. However, directly comparing the present results to these previous studies is somewhat difficult due to methodological differences. Additionally, comparisons between the SI and the OCRA Checklist are rare, and SI comparisons have predominantly been made using the more complex OCRA Index.

Table 1. Strain Index and OCRA Checklist exposure classification criteria used for inter-method reliability analyses.

\begin{tabular}{lcc} 
SI & Risk index classification criteria & \\
& OCRA \\
$<3$ & No risk & $<7.6$ \\
$3-6.9$ & Moderate risk* & $7.6-14$ \\
\hline$\geq 7$ & High Risk & $\geq 14.1$ \\
\hline${ }^{*}$ Moderate risk indicates a magnitude of exposure that may elevate a worker's risk of developing an \\
upper extremity disorder, and employer action is recommended to reduce the exposure.
\end{tabular}

Table 2. Distribution of SI and OCRA Checklist risk classifications for 42 upper extremity cheese manufacturing job exposures to physical MSD risk factors.

\begin{tabular}{|c|c|c|c|c|}
\hline $\begin{array}{l}\text { SI } \\
\text { No. Exposures }\end{array}$ & OCRA & Risk index classification criteria & & No. exposures \\
\hline 1 & $<3$ & No risk & $<7.6$ & 3 \\
\hline 15 & $3-6.9$ & Moderate risk & $7.6-14$ & 18 \\
\hline 26 & $\geq 7$ & High Risk & $\geq 14.1$ & 21 \\
\hline
\end{tabular}




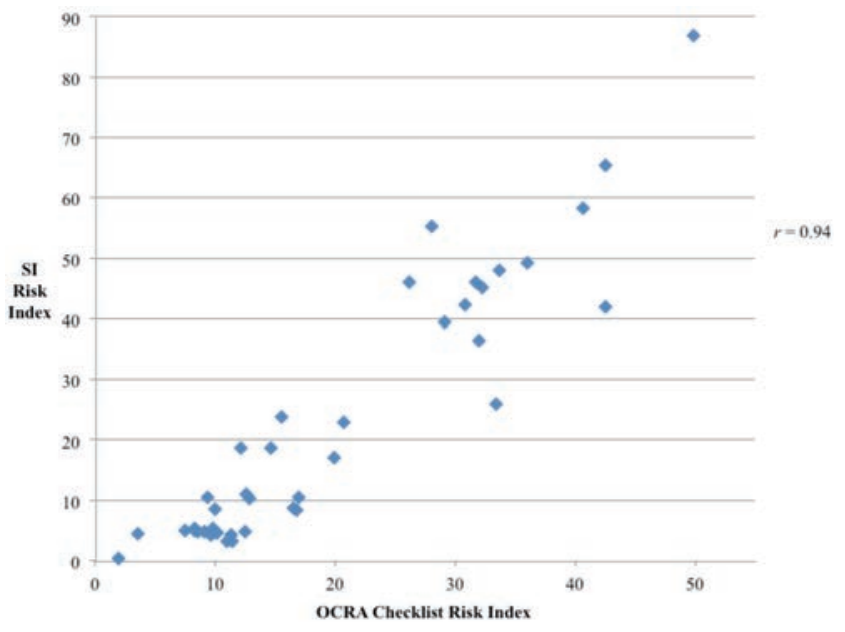

Figure 1. Distribution of SI and OCRA Checklist risk indexes. The Pearson product-moment correlation coefficient $(r)$ was 0.94 .

Apostoli et al., (2004) used the SI and OCRA Checklist to assess 12 repetitive job exposures using a similar risk classification criterion to that of the present study. The authors observed a relatively low proportion of overall agreement $\left(p_{o}=41.7 \%\right)$. Of the seven jobs that were classified differently by the two methods, the SI classified them all at a higher level or risk than did the OCRA Checklist. The present study found overall agreement to be double that of Apostoli's et al., results, but the same trend in systematic bias toward higher SI ratings was observed.

Chiasson et al., (2012) reported $60.1 \%$ proportion of overall agreement between the OCRA Index and SI risk classifications, and they reported a weak to fair correlation between continuous risk indexes $(r=0.32)$. The authors evaluated 167 different job exposures in a variety of industries. The cheese manufacturing jobs evaluated in the present study exhibited much shorter cycle times on average (mean $=41.5$ seconds) than those in Chaisson's et al., study. Inter-method reliability may be strong for SI and OCRA Checklist assessments performed in one industry and weak for those performed in a very different industry.

\section{Conclusions}

The agreement and strength of association between SI and OCRA Checklist risk classifications was strong for the 42 upper limb cheese manufacturing exposures. Further, the correlation between continuous risk indexes was very high $(r=0.94)$. Therefore, inter-method reliability was described as good for mean SI and OCRA Checklist assessments of repetitive cheese manufacturing jobs. Both the SI and the OCRA Checklist appear to be reliable measures of exposure to MSD risk factors in cheese manufacturing. For evaluating physical risk in the agrofood industry, either assessment may be appropriate depending on the needs of the evaluation, the type of work performed, and the experience of the raters. However, the SI is specific to disorders of the distal upper limb and perhaps most useful for assessing risk in work primarily involving the wrist and fingers. The OCRA Checklist may be more appropriate when assessing tasks that require extended periods of reaching and shoulder activity, such as picking fruit or harvesting olives. For jobs that expose agro-food industry workers to repetitive upper extremity movements and forces, either the SI or the OCRA Checklist can be used to reliably quantify physical exposure to MSD risk factors.

\section{References}

Apostoli, P., Sala, E., Gullino, A. \& Romano, C., 2004. [comparative analysis of the use of 4 methods in the evaluation of the biomechanical risk to the upper limb]. Giornale italiano di medicina del lavoro ed ergonomia, 26 (3), 223-241.

Chiasson, M.E., Imbeau, D., Aubry, K. \& Delisle, A., 2012. Comparing the results of eight methods used to evaluate risk factors associated with musculoskeletal disorders. International Journal of Industrial Ergonomics, 42 (5), 478-488.

Colombini, D., Occhipinti, E., Cairoli, S. \& Baracco, A., 2000. [proposal and preliminary validation of a check-list for the assessment of occupational exposure to repetitive movements of the upper limbs]. Medicina Del Lavoro, 91 (5), 470-485.

Colombini, D., Occhipinti, E., Cerbai, M., Battevi, N. \& Placci, M., 2011. [updating of application procedures and criteria for ocra checklist]. Medicina Del Lavoro, 102 (1), 1-39.

Colombini, D., Occhipinti, E. \& Grieco, A., 2002. Risk assessment and management of repetitive movements and exertions of upper limbs job analysis, ocra risk indices, prevention strategies and design principles 0xford: Elsevier.

Jones, T. \& Kumar, S., 2010. Comparison of ergonomic risk assessment output in four sawmill jobs. International Journal of Occupational Safety and Ergonomics, 16 (1), 105-111.

Landis, J.R. \& Koch, G.G., 1977. Measurement of observer agreement for categorical data. Biometrics, 33 (1), 159-174.

Moore, J.S. \& Garg, A., 1995. The strain index - a proposed method to analyze jobs for risk of distal upper extremity disorders. American Industrial Hygiene Association Journal, 56 (5), 443-458.

Spielholz, P., Bao, S., Howard, N., Silverstein, B., Fan, J., Smith, C. \& Salazar, C., 2008. Reliability and validity assessment of the hand activity level threshold limit value and strain index using expert ratings of mono-task jobs. Journal of Occupational \& Environmental Hygiene, 5 (4), 250-257.

Stephens, J.P., Vos, G.A., Stevens, E.M. \& Moore, J.S., 2006. Test-retest repeatability of the strain index. Applied Ergonomics, 37 (3), 275281.

Stevens, E.M., Vos, G.A., Stephens, J.P. \& Moore, J.S., 2004. Inter-rater reliability of the strain index. Journal of Occupational \& Environmental Hygiene, 1 (11), 745-751. 